\title{
Variación de alelos del gen receptor de dopamina DRD4 en escolares chilenos de diferente origen étnico y su relación con riesgo de déficit atencional/hiperactividad
}

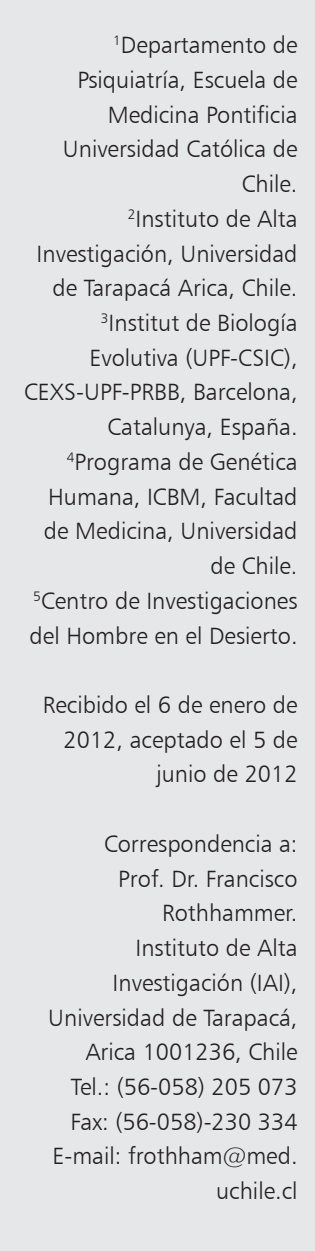

\author{
PAULA ROTHHAMMER ${ }^{1}$, LIZA PAZ LAGOS ${ }^{2}$, \\ YOLANDA ESPINOSA-PARRILLA ${ }^{2,3}$, FRANCISCO ABOITIZ1, \\ FRANCISCO ROTHHAMMER ${ }^{2,4,5}$
}

Background: Worldwide diversity of alleles of D4 receptor gene (DRD4), linked to attention deficit hyperactivity disorder (ADHD), is mostly the result of length and single nucleotide polymorphisms in a 48-bp tandem repeat (VNTR). Alleles containing from two $(2 R)$ to eleven $(11 R)$ repeats have been identified. The most common are $4 R, 7 R$ and 2R. Aim: To study the association of ADHD risk with DRD4 genotypes in Chilean students. Subjects and Methods: ADHD risk data were obtained through the abbreviated Conner's Scale for School Teachers in 66 Aymara children (11 cases and 55 controls), 91 Rapa-Nui children (60 cases ad 31 controls) and 96 children from a mixed urban population from Santiago (51 cases and 45 controls). DNA extracted from saliva was amplified by polymerase chain reaction (PCR) to genotype the DRD4 VNTR. Results: The distribution of DRD4 alleles reveals that, beneath the $4 R$ allele, $7 R$ exhibits the second highest frequencies in Aymara and Santiago children. In Polynesian children, $2 R$ ranks after $4 R$. A statistically significant association between $A D H D$ risk and $2 R / 4 R$ genotype was identified in Polynesian children ( $p<0.05$; odds ratio $=3.7$ ). Conclusions: Different DRD4 genotypes are associated with $A D H D$ phenotype in Chilean populations, probably as a consequence of their initial colonization history.

(Rev Med Chile 2012; 140: 1276-1281).

Key words: Attention deficit disorder with hyperactivity; Child; Dopamine D4; Receptors.

E 1 trastorno de déficit atencional/hiperactividad (TDAH) es una condición de alta prevalencia en numerosas poblaciones a nivel mundial que se caracteriza por un patrón persistente de falta de atención y/o hiperactividadimpulsividad. Investigaciones relacionadas con el TDAH son de particular importancia debido a su relación con conductas que inciden en el rendimiento escolar y la posibilidad de ser és- tas tratadas si se diagnostican tempranamente. El TDAH se ha asociado con alelos del gen del receptor de dopamina DRD4, ubicado cerca del telómero del cromosoma $11 \mathrm{p} 15.5^{1-4}$. La diversidad genética encontrada en este gen es principalmente el resultado de la longitud y de la presencia de polimorfismos de nucleótido único (SNP) en una repetición de $48 \mathrm{pb}$ en tándem (VNTR), ubicada en el exón III. Alelos de DRD4 conteniendo entre 
dos $(2 \mathrm{R})$ y once $(11 \mathrm{R})$ repeticiones con frecuencias que varían considerablemente a nivel mundial se han descrito. El alelo 4R presenta la frecuencia media global más elevada (64\%), seguido por el alelo 7R (media global del 20\%), siendo su frecuencia relativamente baja en Europa, casi ausente en Asia y muy alta en América. El tercer alelo más común es $2 \mathrm{R}$ (media global de $8 \%$ ), mostrando frecuencias bajas en América (3\%), valores ligeramente más altos en Europa (10\%) y frecuencias moderadamente altas en Asia y en el Pacífico $(18 \%)^{5}$. Estos tres alelos representan más de $90 \%$ de la variación genética global observada para DRD4 siendo $4 R / 7 R$ el genotipo que otorga mayor predisposición en América y Europa y $2 R / 4 R$ en $\mathrm{Asia}^{2-5}$. Según estimaciones basadas en comparaciones intra-alélicas en 18 posiciones de alta heterocigosidad en el gen DRD4, el alelo más frecuente, 4R, seria entre 5 y 10 veces más antiguo que $7 \mathrm{R}$, el cual habría surgido probablemente por mutación de 4R hace unos 40.000-50.000 años. El alelo $2 \mathrm{R}$, en cambio, habría surgido más tarde por un simple evento de recombinación / mutación de $4 \mathrm{R}$ y $7 \mathrm{R}^{6,7}$. Los diversos alelos de DRD4 presentan diferentes capacidades para reducir los niveles de adenosín monofosfato cíclico (AMPc), siendo $4 \mathrm{R}$ el más eficiente, seguido de $2 \mathrm{R}$ y $7 \mathrm{R}^{8}$. Por otro lado, los receptores DRD4 actuarían como inhibidores de la activación neuronal en la corteza pre-frontal ${ }^{9,10}$ planteándose una posible relación funcional que implicaría la existencia de una ventaja selectiva de los heterocigotos $4 R / 7 R$ y $2 R / 7 R^{11}$. La distribución geográfica y la importancia funcional de los alelos $2 \mathrm{R}$ y $7 \mathrm{R}$ han dado origen a una reciente polémica metodológica tanto sobre la validez de una posible asociación de $2 \mathrm{R}$ con TDAH en poblaciones asiáticas en las que 7R es casi inexistente, como en la forma en la que los diferentes alelos de DRD4 deben ser clasificados con el fin de obtener resultados significativos y asociaciones genéticas reproducibles ${ }^{11,12}$.

En un trabajo previo ${ }^{13}$ realizado a nivel poblacional informamos sobre la asociación entre riesgo de TDAH y genotipos de DRD4 en escolares chilenos con diferente origen genético. Este trabajo tiene por objetivo validar nuestros resultados anteriores realizando un análisis a nivel individual. La variación interpoblacional en la asociación de alelos de DRD4 con un fenotipo similar (TDAH), podría tener relevancia para comprender las bases funcionales del TDAH.

\section{Material y Método}

Como parte de un estudio previo sobre aspectos genéticos de $\mathrm{TDAH}^{13}$, se obtuvieron estimadores de riesgo de TDAH en escolares chilenos de origen aymara, de origen Rapa-Nui y de la población mixta de Santiago de Chile mediante la aplicación por parte de maestros de escuela del cuestionario de conducta de Conners para profesores ${ }^{14}$. Este cuestionario que utiliza criterios del manual estadístico de diagnóstico DSM-IV, se aplicó para puntuar el riesgo total de TDAH. De acuerdo a la experiencia lograda por nuestro grupo de trabajo ${ }^{15}$, el puntaje de 15 fue elegido como punto de corte para ser clasificado un sujeto de estudio como caso.

Para la tipificación genética se utilizó un método no invasivo aislando $\mathrm{ADN}$ genómico a partir de saliva utilizando el kit "Oragene ${ }^{\circledR} \cdot D N A$ sample collection kit (DNA Genotek, Ontario, Canada)" según el protocol de extracción manual de ADN proporcionado por el fabricante. Los genotipos para el polimorfismo VNTR del locus DRD4 se determinaron mediante la técnica de amplificación por reacción en cadena de la polimerasa (PCR) utilizando cebadores previamente descritos ${ }^{15} \mathrm{y}$ $10 \%$ DMSO. Tras un primer paso de incubación a $94{ }^{\circ} \mathrm{C}$ durante $2 \mathrm{~min}$, se realizaron 35 ciclos de PCR $\left(95^{\circ} \mathrm{C}\right.$ durante $30 \mathrm{~s}, 61^{\circ} \mathrm{C}$ durante 30 s y $72^{\circ} \mathrm{C}$ durante 1 min $30 \mathrm{~s}$ ) y 5 min a $72{ }^{\circ} \mathrm{C}$ de extensión final. La determinación de los genotipos se realizó directamente a partir de la visualización de los productos de PCR separados durante $6 \mathrm{~h}$ a $40 \mathrm{~V}$ en un gel de agarosa al $2 \%$ y posteriormente teñidos con GelRedTM (Biotium, Hayward, Estados Unidos de Norteamérica).

La significación entre las diferencias en los estimadores de riesgo de TDAH y el grado de asociación con los genotipos de DRD4 se determinó mediante $\chi^{2}$. La razón de verosimilitud ("Odds Ratio”, OR) se estimó siguiendo el método tradicional descrito por Woolf, $1956^{16}$ y Haldane, $1956^{17}$. Como consecuencia de las dificultades en la obtención de muestras en lugares apartados y grupos poblacionales reducidos, el número de individuos analizado es en algunos casos relativamente pequeño, condición que podría haber incidido en la significación estadística de algunas comparaciones.

Este trabajo se inscribe dentro de una línea de investigación iniciada hace más de una década a 
través de una colaboración entre el Departamento de Psiquiatría de la Facultad de Medicina de la Pontificia Universidad Católica de Chile y el Programa de Genética Humana del ICBM de la Facultad de Medicina de la Universidad de Chile, habiéndose realizado el muestreo poblacional durante distintas fases del estudio. Los protocolos fueron aprobados por el Comité de Ética de la Facultad de Medicina de la Universidad de Chile y están de acuerdo con las normas establecidas en la declaración de Helsinki de 1964.

\section{Resultados}

La distribución de la muestra total según género no resultó ser significativamente diferente y, en consecuencia, el estudio de asociación se realizó agrupando ambos géneros en un mismo grupo. En cuanto al riesgo de TDAH, 8,33\% de los escolares de origen aymara, 9,73\% de los escolares de Santiago de Chile y 20,79\% de los escolares de origen Rapa-Nui tuvieron puntajes iguales o superiores a 15 siendo considerados como casos. Los demás escolares se consideraron controles. Las diferencias entre los estimadores de riesgo de TDAH resultaron ser significativas $\left(\chi^{2}=23,38\right.$ $\mathrm{p}=0,0001)$.

Los genotipos para el VNTR de DRD4 de los escolares aymara, de Santiago de Chile y Rapa-Nui se muestran en la Tabla 1. No se encontró asociación genética entre el VNTR de DRD4 y riesgo de TDAH en los escolares aymara. En el caso de

Tabla 1. Asociación entre el riesgo a TDAH y los genotipos de DRD4 en escolares chilenos

\begin{tabular}{|c|c|c|c|c|c|c|c|c|}
\hline \multicolumn{9}{|c|}{ Tabla 1a. Escolares Aymara de los Andes } \\
\hline & 2R/4R & 3R/4R & 4R/4R & 4R/6R & 4R/7R & 6R/7R & 7R/7R & Total \\
\hline TDAH & 0,00 & 0,18 & 0,36 & 0,09 & 0,18 & 0,00 & 0,18 & \\
\hline N & 0 & 2 & 4 & 1 & 2 & 0 & 2 & 11 \\
\hline Controles & 0,18 & 0,18 & 0,31 & 0,09 & 0,34 & 0,02 & 0,20 & \\
\hline $\mathrm{N}$ & 1 & 1 & 17 & 5 & 19 & 1 & 11 & 55 \\
\hline Total & 0,12 & 0,03 & 0,22 & 0,06 & 0,09 & 0,32 & 0,02 & \\
\hline $\mathrm{N}$ & 1 & 3 & 21 & 6 & 21 & 1 & 13 & 66 \\
\hline
\end{tabular}

\begin{tabular}{|c|c|c|c|c|c|c|c|c|c|c|c|}
\hline & & & Tabla 1 & o. Escol & res Rap & -Nui de & sla de P & scua & & & \\
\hline & $2 R / 2 R$ & $2 R / 3 R$ & $2 R / 4 R$ & $3 R / 3 R$ & $3 R / 4 R$ & $4 R / 4 R$ & $4 R / 5 R$ & 4R/7R & $5 R / 7 R$ & 7R/7R & Total \\
\hline TDAH & 0,12 & 0,03 & 0,28 & 0,05 & 0,05 & 0,30 & 0,02 & 0,10 & 0,02 & 0,02 & \\
\hline $\mathrm{N}$ & 7 & 3 & 17 & 3 & 3 & 18 & 1 & 6 & 1 & 1 & 60 \\
\hline Controles & 0,13 & 0,00 & 0,10 & 0,07 & 0,16 & 0,36 & 0,03 & 0,07 & 0,00 & 0,10 & \\
\hline $\mathrm{N}$ & 4 & 0 & 3 & 2 & 5 & 11 & 1 & 2 & 0 & 3 & 31 \\
\hline Total & 0,12 & 0,03 & 0,22 & 0,06 & 0,09 & 0,32 & 0,02 & 0,09 & 0,01 & 0,04 & \\
\hline $\mathrm{N}$ & 11 & 3 & 20 & 5 & 8 & 29 & 2 & 8 & 1 & 4 & 91 \\
\hline
\end{tabular}

\begin{tabular}{|c|c|c|c|c|c|c|c|c|}
\hline \multicolumn{9}{|c|}{ Tabla 1c. Escolares de Santiago de Chile } \\
\hline & 2R/4R & 2R/7R & 4R/4R & 4R/5R & 4R/6R & 4R/7R & 7R/7R & Total \\
\hline TDAH & 0,08 & 0,02 & 0,51 & 0,02 & 0,10 & 0,25 & 0,02 & \\
\hline N & 4 & 1 & 26 & 1 & 5 & 13 & 1 & 51 \\
\hline Controles & 0,09 & 0,02 & 0,53 & 0,04 & 0,11 & 0,20 & 0,00 & \\
\hline N & 4 & 1 & 24 & 2 & 5 & 9 & 0 & 45 \\
\hline Total & 0,08 & 0,02 & 0,52 & 0,03 & 0,10 & 0,23 & 0,01 & \\
\hline N & 8 & 2 & 50 & 3 & 10 & 22 & 1 & 96 \\
\hline
\end{tabular}


los escolares de Santiago de Chile, se observó una tendencia estadísticamente no significativa a la asociación entre riesgo de TDAH y el genotipo $4 R / 7 R$. En cambio, se encontró una asociación entre riesgo de TDAH y el genotipo $2 R / 4 R(\mathrm{p}<0,05$, $\mathrm{OR}=3,7)$ en escolares Rapa-Nui. La frecuencia relativamente alta de $2 \mathrm{R}$ en escolares Rapa-Nui concuerda con resultados obtenidos previamente en poblaciones polinésicas específicamente en los habitantes de Samoa y Micronesia ${ }^{5}$.

\section{Discusión}

Nuestros resultados muestran diferencias significativas entre las estimaciones de riesgo de TDAH obtenidas en escolares chilenos de diferente origen genético y geográfico, mostrando los escolares Rapa-Nui el puntaje más elevado. Puntajes altos de riesgo de TDAH se asocian de manera estadísticamente significativa al genotipo $2 R / 4 R$ en este grupo $(\mathrm{OR}=3,7)$, no alcanzando niveles de significación estadística la asociación con $4 R / 7 R$. Este resultado replica una tendencia observada en Asia ${ }^{18}$. En los escolares aymara, el genotipo $4 R / 7 R$ presenta una frecuencia relativamente alta, aunque no está vinculado al riesgo de TDAH; en cambio el genotipo $2 R / 4 R$ presenta una frecuencia muy baja. En los escolares de Santiago de Chile, $2 R / 4 R$ y $4 R / 7 R$ están presentes en baja frecuencia y $4 R / 7 R$ muestra una tendencia no significativa a estar asociado con riesgo de TDAH. El alelo 4R es el más común en las tres poblaciones estudiadas (Tabla 2), seguido por 7R en escolares aymaras $y$ poblaciones urbanas y por $2 \mathrm{R}$ en estudiantes Rapa-Nui.

Es posible que la selección natural haya actuado sobre el gen DRD4, incrementando la frecuen- cia del alelo 7R durante el desplazamiento, hace 40.000 años, de los primeros humanos modernos desde África hacia Europa y Asia, como resultado de una mayor capacidad de 7R para reducir los niveles de adenosín monofosfato cíclico (AMPc) y en consecuencia aumentar el comportamiento impulsivo de los portadores ${ }^{7}$. Hemos señalado que $2 \mathrm{R}$ apareció después de $7 \mathrm{R}$ y presenta una respuesta intermedia al AMPc, entre $4 \mathrm{R}$ y $7 \mathrm{R}$. Teóricamente esta característica funcional podría haber sido aún más ventajosa que aquella otorgada a los portadores de $7 \mathrm{R}$, lo que explicaría una mayor frecuencia del alelo $2 \mathrm{R}$ y una menor frecuencia el alelo 7R en Asia oriental y meridional. Recientemente se ha demostrado que en niños chinos Han el alelo 2R se asocia a $\operatorname{TDAH}^{18} \mathrm{y}$, el caso de individuos coreanos y filipinos, $2 \mathrm{R}$ se ha asociado al rasgo de temperamento de búsqueda de novedad (novelty seeking) que ha sido a menudo relacionado con el TDAH ${ }^{11}$. Por otra parte, la frecuencia de $2 \mathrm{R}$ en sujetos chinos con TDAH aumenta de una manera parecida al incremento que se observa para el alelo 7R en casos europeos de $\mathrm{TDAH}^{18}$.

Sin duda, la gran diferencia en la frecuencia de $7 \mathrm{R}$ entre poblaciones asiáticas y amerindias ${ }^{5}$ es intrigante tomando en consideración que América se pobló a partir de Asia. De acuerdo con nuestros estudios, la frecuencia del alelo 7R en las poblaciones aymara es alta (27-38\%) pero inferior a la media mundial que se ha publicado para poblaciones amerindias (48\%), cuyo cálculo incluye, principalmente, poblaciones no andinas de América del Sur ${ }^{5}$. En consecuencia, la diferencia de frecuencia del alelo $7 \mathrm{R}$ que se observa entre las poblaciones de Asia y América podría haber sido inicialmente mucho más pequeña. La ocurrencia durante la colonización de América

Tabla 2. Alelos del VNTR de DRD4 en escolares de los Andes, Isla de Pascua y Santiago de Chile

\begin{tabular}{|llllllllll|}
\hline \multirow{2}{*}{ Andes } & $\mathbf{2 R}$ & $\mathbf{3 R}$ & $\mathbf{4 R}$ & $\mathbf{5 R}$ & $\mathbf{6 R}$ & $\mathbf{7 R}$ & $\mathbf{N}$ \\
& Casos & 0,00 & 0,09 & 0,59 & 0,00 & 0,04 & 0,27 & 11 \\
\multirow{2}{*}{ Isla de Pascua } & Controles & 0,01 & 0,01 & 0,54 & 0,00 & 0,05 & 0,38 & 55 \\
& Casos & 0.28 & 0,10 & 0,53 & 0,02 & 0,00 & 0,07 & 60 \\
\multirow{2}{*}{ Santiago } & Controles & 0,18 & 0,14 & 0,53 & 0,02 & 0,00 & 0,13 & 31 \\
& Casos & 0,05 & 0,00 & 0,74 & 0,01 & 0,05 & 0,15 & 51 \\
& Controles & 0,05 & 0,00 & 0,76 & 0,02 & 0,06 & 0,11 & 45 \\
\hline
\end{tabular}


de efecto fundador no se puede tampoco excluir como explicación adicional. En efecto, recientemente se han analizado los patrones de diversidad genética en el ADN nuclear y ADN mitocondrial entre poblaciones americanas originarias; dichos estudios evidencian una historia demográfica heterogénea en América del Sur, con poblaciones andinas que divergen antes y con tamaños efectivos mayores ${ }^{19,20}$.

Hemos señalado previamente que Isla de Pascua fue poblada a partir de Asia alrededor del año 2000 A.P., unos 11.500 años más tarde que América $^{21}$. Este tiempo es suficiente como para que tuvieran lugar cambios significativos en las frecuencias génicas de DRD4.

Concluyendo, los niños aymara no demuestran presentar una asociación entre alelos del gen DRD4 y riesgo deTDAH. Los niños Rapa-Nui podrían tener un riesgo más alto de TDAH debido a la presencia de ciertos alelos menos frecuentes $\mathrm{o}$ ausentes en otras poblaciones chilenas, como el alelo R2. Este alelo confiere un mayor riesgo de TDAH que R7 en poblaciones asiáticas a partir de las cuales se originaron los Rapa-Nui. Los escolares de Santiago presentan una asociación entre $4 R / 7 R$ y riesgo de TDAH, pero esta no alcanza significación estadística, Sin embargo, hemos señalado que el TDAH tiene un componente genético alto ${ }^{1}$ y que el gen DRD4 ha sido predominantemente vinculado a su etiología. Es entonces de interés que en distintas poblaciones chilenas diferentes alelos del gen DRD4 se encuentran asociados al mismo fenotipo (TDAH). Este hallazgo podría tener consecuencias para el tratamiento del TDAH debido a que los alelos implicados son funcionalmente diferentes.

Agradecimientos: Agradecemos a los profesores de los colegios de Isla de Pascua, Región de Arica-Parinacota y Región Metropolitana por su entusiasta y desinteresada participación en el presente estudio. Agradecemos también a D. Comas, M. Vallé, C.E. Gómez y H. Henríquez por su apoyo técnico y científico y a los revisores anónimos que contribuyeron a mejorar este manuscrito. Este trabajo ha sido financiado a través de fondos otorgados por los Proyecto Fondecyt \# 1080219, el Convenio de Desempeño UTA/Mecesup 2 y el programa de la Unión Europea "European Union Seventh Framework Programme" (PIOFGA-2009-236836).

\section{Referencias}

1. Faraone SV, Perlis DH, Doyle AE, Smoller JW, Goralnick JJ, Holmgren MA, et al. Molecular genetics of attention deficit/hyperactivity disorder. Biol Psychiatry 2005; 57 (11): 1313-23.

2. LaHoste GJ, Swanson JM, Wigal SB, Glabe C, Wigal T, King N, et al. Dopamine D4 receptor gene polymorphism is associated with attention deficit hyperactivity disorder. Mol Psychiatry 1996; 1 (2): 121-4.

3. Swanson JM, Flodman P, Kennedy J, Spence MA, Moyzis R, Schuck S, et al. Dopamine genes and ADHD. Neurosci Behav Rev 2000; 24 (1): 21-5.

4. Faraone SV, Doyle AE, Mick E, Biederman J. Metaanalysis of the association between the 7-repeat of the dopamine D (4) receptor gene and attention deficit hyperactivity disorder. Am J Psychiatry 2001; 158 (7): 1052-7.

5. Chang FM, Kidd JR, Livak KJ, Pakstis AJ, Kidd KK. The world-wide distribution of allele frequencies at the human dopamine D4 receptor locus. Hum Genet 1996; 98 (1): 91-101.

6. Ding YC, Chi HC, Grady DL, Morishima A, Kidd JR, Kidd KK, et al. Evidence of positive selection acting at the human dopamine receptor D4 gene locus. Proc Natl Acad Sci USA 2002; 99 (1): 309-14.

7. Wang E, Ding YC, Flodman P, Kidd JR, Kidd KK, Grady $\mathrm{DL}$, et al. The genetic architecture of selection at the human dopamine receptor D4 (DRD4) gene locus. Am J Hum Genet 2004; 74 (5): 931-44.

8. Asghari V, Sanyal S, Buchwaldt S, Paterson A, Jovanovic V, Van Tol H. Modulation of intracellular cyclic AMP levels by different human dopamine D4 receptor variants. J Neurochem 1995; 65 (3): 1157-65.

9. Oak JN, Oldenhof J, Van Tol HH. The dopamine D (4) receptor: one decade of research. Eur J Pharmacol 2000; 405 (1-3): 303-27.

10. Rubinstein M, Cepeda C, Hurst RS, Flores-Hernández J, Ariano MA, Falzone TL, et al. Dopamine D4 receptordeficient mice display cortical hyperexcitability. J Neurosci 2001; 21 (11): 3756-63.

11. Reist C, Ozdemir V, Wang E, Hashemzadeh M, Mee S, Moyzis R. Novelty seeking and the dopamine D4 receptor gene (DRD4) revisited in Asians: haplotype characterization and relevance of the 2-repeat allele. Am J Med Genet B Neuropsychiatr Genet 2007; 144B (4): 453-7.

12. Lee HJ. Comments on "Novelty seeking and the dopamine D4 receptor gene (DRD4) revisited in Asians: Haplotype characterization and relevance of the 2-repeat allele" by C. Reist et al. Am J Med Genet B Neuropsy- 
chiatr Genet 2007; 144 (4): 453-457. Am J Med Genet B Neuropsychiatr Genet 2009; 150 B (1): 151-2.

13. Lagos LP, Silva C, Rothhammer P, Carrasco X, Llop E, Aboitiz F, et al. Risk of attention deficit/hyperactivity disorder in Aymara and Rapa-Nui school children: Association with dopaminergic system polymorphisms. Rev Med Chile 2011; 139 (5): 600-5.

14. Conners CK. Conners Rating Scales-Revised.Technical Manual. Toronto: Multi-Health Systems. 1997.

15. Holmes J, Payton A, Barrett JH, Hever T, Fitzpatrick $\mathrm{H}$, Trumper AL, et al. A family-based and case-control association study of the dopamine D4 receptor gene and dopamine transporter gene in attention deficit hyperactivity disorder. Mol Psychiatry 2000; 5 (5): 523-30.

16. Woolf B. On estimating the relation between blood group and disease. Ann Hum Genet 1955; 19: 251-3.

17. Haldane JBS. The estimation and significance of the logarithm of a ratio of frequencies. Ann Hum Genet
1956; 20: 309-11.

18. Leung PW, Lee CC, Hung SF, Ho TP, Tang CP, Kwong $\mathrm{SL}$, et al. Dopamine receptor D4 (DRD4) gene in Han Chinese children with attention-deficit/hyperactivity disorder (ADHD): increased prevalence of the 2-repeat allele. Am J Med Genet B Neuropsychiatr Genet 2005; 133 B (1): 54-6.

19. Wang ET, Moyzis RK. Genetic evidence for ongoing balanced selection at human DNA repair genes ERCC8, FANCC, and RAD51C. Mutat Res 2007; 616 (1-2): 16574.

20. Yang NN, Mazières $S$, Bravi $C$, Ray $N$, Wang $S$, Burley $\mathrm{MW}$, et al. Contrasting patterns of nuclear and mtDNA diversity in Native American populations. Ann Hum Gene 2010; 74(6): 525-38.

21. Rothhammer F, Dillehay TD. The late Pleistocene colonization of South America: an interdisciplinary perspective. Ann Hum Genet 2009; 73 (5): 540-9. 